\title{
Enthalpy of solution of terfenadine in ethanol/water mixtures
}

\author{
João Canotilho ${ }^{\mathrm{a}}$, Felisbela S. Costa ${ }^{\mathrm{b}}$, Adriano T. Sousa ${ }^{\mathrm{a}}$, J. Simões Redinha ${ }^{\mathrm{c}}$, \\ M. Luísa P. Leitão, ${ }^{\mathrm{c},}$ \\ ${ }^{a}$ Laboratório de Galénica e Tecnologia Farmacêutica, Faculdade de Farmácia, Universidade de Coimbra, 3000 Coimbra, Portugal \\ ${ }^{\mathrm{b}}$ Laboratório de Métodos Instrumentais de Análise, Faculdade de Farmácia, Universidade de Coimbra, 3000 Coimbra, Portugal \\ ${ }^{\mathrm{c}}$ Departamento de Química, Faculdade de Ciências e Technologia, Universidade de Coimbra, 3000 Coimbra, Portugal
}

Accepted 23 August 1999

\begin{abstract}
The enthalpy of solution of terfenadine in ethanol/water mixtures, $0-20.5 \mathrm{wt} . \%$ of water was determined by calorimetry. A Sudden increase of $1-2 \mathrm{~kJ} \mathrm{~mol}^{-1}$ in the enthalpy at a concentration value around $0.01 \mathrm{~mol} \mathrm{~kg}^{-1}$ is observed. This step in the enthalpy is interpreted as due to solute n-mer aggregates formation. The solubility of terfenadine in the cosolvent systems used in the calorimetric studies was determined. (C) 2000 Elsevier Science B.V. All rights reserved.
\end{abstract}

Keywords: Crystal design; Crystallization; Polymorphism; Solubility; Solute self-aggregation; Solution enthalpy; Terfenadine

\section{Introduction}

It has been admitted that terfenadine, $\alpha$-[4-(1,1dimethylethyl) phenyl]-4-(hydroxy diphenylmethyl)1-piperidine butanol, gives rise to different polymorphic forms when obtained from different solvents or in different conditions [1-4]. Solution calorimetry is being used here with a two fold objective: to get information on the solid structure obtained by crystallization and to understand the role of the solvent in these methods of preparation [5]. The results obtained show that enthalpy of solution is effective for these objectives.

The present paper is directed mainly to the interpretation of solute-solute interactions of terfenadine in solution. On the grounds that solute association in solution plays an important role in the structure of the

\footnotetext{
${ }^{*}$ Corresponding author. Fax: +351-39-827703.

E-mail address: mlleitao@cygnus.ci.uc.pt (M. P. Leitão).
}

solid phase separating from the liquid when the saturation is reached, the study of the interactions involving the solute is crucial for the interpretation of polymorphism and in crystal engineering. Ethanol/ water mixtures were the solvents used. Cosolvent systems, namely ethanol/water, are often used as crystallization media for drugs. On the other hand from the results obtained for these mixtures one can infer the behavior of the solute in water. Aqueous solutions cannot be directly studied due to the very low solubility of terfenadine in this solvent.

\section{Experimental}

The terfenadine sample used in this study was obtained by crystallization from an ethanol/water mixture $(80: 20, \mathrm{v} / \mathrm{v})$ saturated at $60^{\circ} \mathrm{C}$. The original material was purchased from Sigma Chemical. The crystallization was performed by keeping the solution 
at room temperature for $24 \mathrm{~h}$. The solid formed was recovered by filtration and dried in a vacuum oven at $40^{\circ} \mathrm{C}$ for $48 \mathrm{~h}$. Ethanol from Merck ( $\geq 99.9 \%$, ACS, Uvasol grade) and water delivered by a Millipore purifier unit were used in the preparation of solvent mixtures.

Cosolvent systems in which the water varies between 0 and 20.5 wt.\% were prepared. Due to the low solubility of the terfenadine in water, solutions of water content above this limit cannot be calorimetrically studied. The apparatus and procedures used for the enthalpy determinations were described previously [5].

For the solubility measurements, approximately $10 \mathrm{ml}$ of terfenadine saturated solutions with an excess of solid were put into $20 \mathrm{ml}$ borosilicate glass vials sealed by a rubber stopper fastened to the mouth of the vial by an aluminium capsule. The vials were placed in a stainless steel basket kept swinging in a water bath at $25^{\circ} \mathrm{C}$ by means a mechanical device. It was observed that after $70 \mathrm{~h}$ equilibrium was surely reached and then a sample of solution was withdrawn for concentration determination. A hypodermic syringe to which a Millipore cartridge microfilter, pore size $0.45 \mu \mathrm{m}$, was adapted, was used for transferring the solution to a certain amount of the previously weighed solvent. The collection of the solution sample was carried out by piercing the rubber stopper. All solutions were prepared by weight and in order to avoid solid formation inside the syringe its temperature was about $10^{\circ}$ above that of the solution. The determination of the concentrations were performed by absorption spectrophotometry using the $\pi^{*} \leftarrow \pi$ electronic transition, $\lambda_{\max }=$ $259.8 \mathrm{~nm}$.

\section{Results}

The results observed for the enthalpy of solution as a function of the concentration for different cosolvent compositions are shown in Fig. 1. The results obtained for the solubility of terfenadine are presented in Table 1. The figures inserted in this table are a mean of seven determinations.

\section{Discussion}

The variation of the solution enthalpy with concentration has an important significance in the context of this work as it is ascribed to solute-solute interactions. In ethanol the variations of $\Delta_{\text {sol }} H$ with $m$ is small

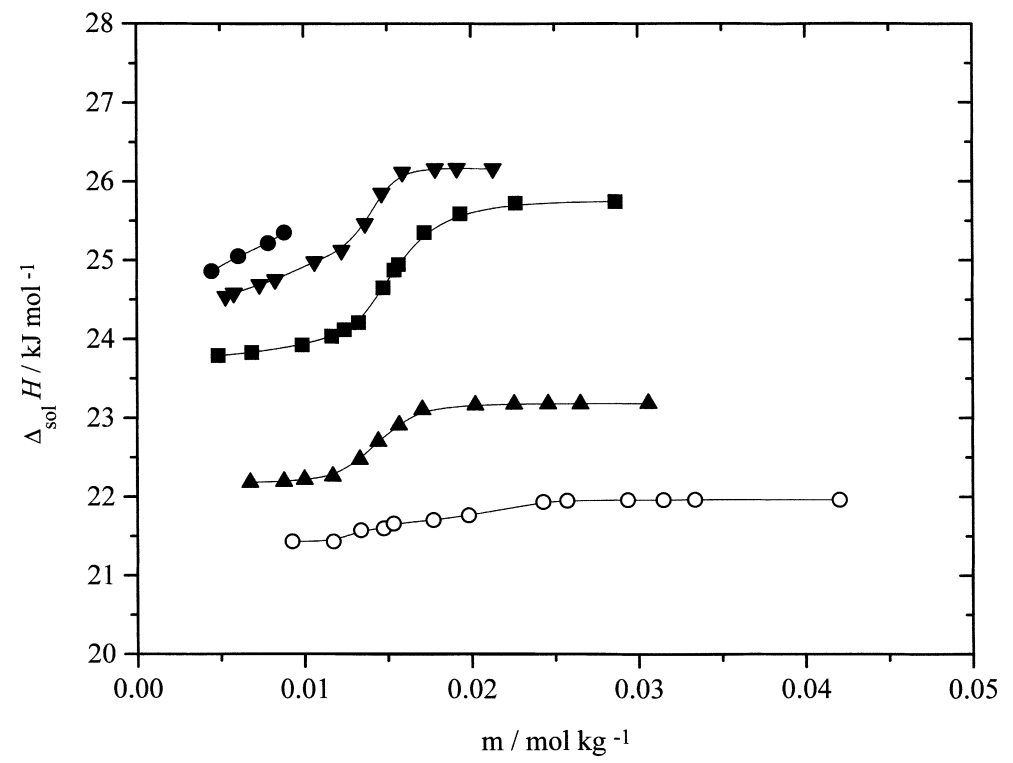

Fig. 1. Enthalpy of solution of terfenadine in ethanol and ethanol/water mixtures at $298.15 \mathrm{~K}$. (O) ethanol;

( $)$ ethanol/water $(90: 10, \mathrm{v} / \mathrm{v})$; ( $\boldsymbol{\nabla})$ ethanol/water $(85.15, \mathrm{v} / \mathrm{v})$; (O) ethanol/water $(80: 20, \mathrm{v} / \mathrm{v})$. 
Table 1

Solubility of terfenadine in ethanol/water cosolvent systems at $298.15 \mathrm{~K}$

\begin{tabular}{ll}
\hline Cosolvent composition & Solubility $\left(\mathrm{mol} \mathrm{kg}^{-1}\right)$ \\
$X_{\text {water }}($ wt. $\%$ water $)$ & \\
\hline $0.000(00.00)$ & $0.071 \pm 0.0017$ \\
$0.145(05.04)$ & $0.049 \pm 0.0013$ \\
$0.264(10.15)$ & $0.035 \pm 0.0019$ \\
$0.363(15.32)$ & $0.0221 \pm 0.00026$ \\
$0.447(20.50)$ & $0.0135 \pm 0.00024$ \\
\hline
\end{tabular}

whereas in the cosolvent with 20.5 wt.\% of water a high value for the slope is found. Between these two limiting patterns a striking feature is observed in $\Delta_{\text {sol }} H$ vs. $m$ curves. As the concentration reaches about $0.01 \mathrm{~mol} \mathrm{~kg}^{-1}$ a sudden increase of the enthalpy with concentration occurs. A step up of $1-2 \mathrm{~kJ} \mathrm{~mol}^{-1}$ in the enthalpy is observed for an increasing of concentration, say, $0.005 \mathrm{~mol} \mathrm{~kg}^{-1}$ in cosolvent systems of water $5-15$ wt. $\%$.

The pattern presented by $\Delta_{\text {sol }} H$ vs. $m$ curves for terfenadine in ethanol/water is rather similar to that observed for some properties of surfactants in aqueous solutions and ascribed to micelle formation [6-8]. Besides a large non-polar part, terfenadine has two $\mathrm{OH}$ groups which can confer to it an amphiphilic behavior. Indeed, the existence of a step in $\Delta_{\text {sol }} H$ vs. $m$ curves seems to give evidence to a self-association of the solute according to the following reaction:

$$
n \mathrm{~T}_{1} \rightarrow \mathrm{T}_{n}
$$

That is, $n$ terfenadine molecules give, by selfassembling, rise to an $n$-mer solute unit. The value of the concentration at which this type of aggregation occurs is called critical aggregation concentration (CAC).

Before CAC being reached a small variation of $\Delta_{\text {sol }} H$ with concentration is observed in the cosolvent of low water content. As water increases in the cosolvent an increase of $\Delta_{\text {sol }} H$ with concentration is observed. These findings show that when the water content is low, in a concentration range up to CAC, terfenadine is present mainly as monomers. In richer water cosolvents a stepwise association, giving aggregates of a small number of solute molecules, takes place. After CAC, $\Delta_{\text {sol }} H$ remains constant as concentration increases. The $\mathrm{n}$-mers formed by co-operative effect of self-assembly are the nucleus of the solid separated from the liquid at higher concentrations. Similarly to what is observed for other systems, the nucleation is a calorimetrically active step whereas the growth leading to the solid phase is calorimetrically silent [9].

It is noteworthy that, in the range of the mixtures studied, CAC is almost independent of the cosolvent composition. Very likely, the capacity for assembling the solute molecules in clusters of high aggregation number is shown provided the cosolvent exhibits a mixed behavior of the two liquids. This happens to the limit $X_{\mathrm{w}} \approx 0.5$ after which the cosolvent behaves towards terfenadine as water [10].

Without details, a typical $\Delta_{\text {sol }} H$ vs. $m$ curve for terfenadine in ethanol/water mixtures consists of three regions: the first whose upper limit is CAC, is characterized by the enthalpy value of $\Delta_{\text {sol }} H^{0}$; the second is a very narrow concentration range around CAC in which a deep step of $\Delta_{\mathrm{sol}} H$ is observed; the third consisting of concentration above CAC is characterized by the maximum value found for $\Delta_{\text {sol }} H$.

This simplified curve, not much different from those obtained for low water content, leads to admit the existence of an ideal solution of monomeric solute molecules for concentrations below CAC and a system consisting of monomers and n-mer aggregates in equilibrium in concentrations above this limit. The aggregates can be considered thermodynamically as a distinct phase which, being in the liquid state, is called a pseudophase. The concept of pseudophase is an approach sometimes used for interpreting the micellization of surfactants [6,11-14].

In the context of this work the introduction of this concept implies that the chemical potential of terfenadine in the pseudophase is not dependent on the concentration. We have no data for Gibbs energy in this respect. Nevertheless, the constancy of $\Delta_{\mathrm{sol}} H$ for concentration above CAC gives some support to the assumption.

The interpretation of the solution process can then, be based in a thermodynamic cycle involving solid terfenadine and the phases just described. The variation of Gibbs energy when one mole of terfenadine is dissolved in a certain solvent originating a dilute solution is given by the following general equation:

$$
\Delta_{\mathrm{sol}} G=\mu_{\mathrm{T}}^{0}(\mathrm{sol})+R T \ln m+\Delta \mu_{\mathrm{T}}^{\mathrm{E}}-\mu_{\mathrm{T}}^{*}(\mathrm{~s}) .
$$


Table 2

Standard thermodynamic properties of solution of terfenadine in ethanol/water cosolvent systems ${ }^{\mathrm{a}}$

\begin{tabular}{|c|c|c|c|c|c|c|c|c|c|}
\hline \multirow{2}{*}{$\begin{array}{l}\text { Solvent } \\
\text { (wt.\% water) }\end{array}$} & \multicolumn{3}{|c|}{$\mathrm{A}(\mathrm{s}) \rightarrow \mathrm{A}_{1}(\mathrm{sol})$} & \multicolumn{3}{|c|}{$n \mathrm{~A}_{1}(\mathrm{sol}) \rightarrow \mathrm{A}_{n}(\mathrm{sol})$} & \multicolumn{3}{|c|}{$\mathrm{A}(\mathrm{s}) \rightarrow \mathrm{A}_{n}$ (sat. sol) } \\
\hline & $\Delta_{\mathrm{sol}} G_{1}^{0}$ & $\Delta_{\text {sol }} H_{1}^{0}$ & $\mathrm{~T} \Delta_{\mathrm{sol}} S_{1}^{0}$ & $\Delta_{\mathrm{ag}} G^{0}$ & $\Delta_{\mathrm{ag}} H^{0}$ & $\mathrm{~T} \Delta_{\mathrm{ag}} S^{0}$ & $\Delta_{\mathrm{sol}} G_{n}^{0}$ & $\Delta_{\mathrm{sol}} H_{n}^{0}$ & $\mathrm{~T} \Delta_{\mathrm{sol}} S_{n}^{0}$ \\
\hline 0 & - & 21.315 & - & - & - & - & 6.280 & 21.794 & 15.694 \\
\hline 5.041 & 18.415 & 22.179 & 3.764 & -11.046 & 0.998 & 12.044 & 7.451 & 23.177 & 16.726 \\
\hline 10.154 & 19.252 & 23.821 & 4.569 & -11.004 & 1.911 & 12.915 & 8.248 & 25.732 & 17.484 \\
\hline 15.316 & 20.312 & 24.620 & 4.308 & -11.927 & 1.591 & 12.518 & 9.385 & 26.211 & 16.826 \\
\hline
\end{tabular}

${ }^{\mathrm{a}}$ Results in $\mathrm{kJ} \mathrm{mol}^{-1}$ at $298.15 \mathrm{~K}$.

T stands for terfenadine and the standard states for the solute chemical potential are infinite dilution and pure solid in solution and in solid phase, respectively. $\Delta \mu_{\mathrm{T}}^{\mathrm{E}}$ is the excess chemical potential for the solute at concentration $m$. Eq. (2) can be written in the form:

$$
\Delta_{\text {sol }} G=\Delta_{\text {sol }} G^{0}+R T \ln m+\Delta \mu_{\mathrm{T}}^{\mathrm{E}} .
$$

A similar expression is obtained for the variation of the enthalpy:

$$
\Delta_{\text {sol }} H=\Delta_{\text {sol }} H^{0}+\Delta H_{\mathrm{T}}^{\mathrm{E}} .
$$

From the results obtained for $\Delta_{\mathrm{sol}} H$ presented in Fig. 1 the values for the enthalpy of the individual processes can be calculated. The enthalpy variation for transferring the solute from the solid phase to an ideal solution of monomers, $\Delta_{\text {sol }} H_{1}^{0}$ is the limiting value of $\Delta_{\text {sol }} H$ for zero concentration; that needed for transferring the solute from the solid phase to the aggregate pseudophase, $\Delta H_{n}^{0}$, is the upper limit of $\Delta_{\text {sol }} H$, which is given by the values corresponding to concentrations above CAC; the transfer of the solute between monomer solution and the n-mer pseudophase, $\Delta_{\mathrm{ag}} H_{n}^{0}$, is the difference between the two other terms. By fitting a Boltzmann sigmoidal equation to the experimental values obtained for $\Delta_{\text {sol }} H$ the three enthalpic contributions were determined.

Gibbs energy terms that can be determined are $\Delta_{\text {sol }} G_{n}^{0}$, and $\Delta_{\mathrm{ag}} G_{n}^{0}$. From the equilibrium, solid/saturated solution, the following equation can be written:

$$
\Delta_{\mathrm{sol}} G_{n}^{0}=\mu_{\mathrm{T}, n}^{0}(\mathrm{ag})-\mu_{\mathrm{T}}^{*}(\mathrm{~s})=-R T \ln S,
$$

where $S$ is the molar solubility. This equation shows that variation of Gibbs energy by transferring one mole of the solute from the solid phase to the saturated solution can be calculated directly from the results obtained for the solubility.
The standard Gibbs energy for the formation of a n-mer unit from monomers according to the model accepted for the aggregates is given by:

$$
\Delta_{\mathrm{ag}} G_{n}^{0}=\left(\mu_{\mathrm{T}, n}^{0}-n \mu_{\mathrm{T} 1}^{0}\right)=n R T \ln m_{1},
$$

where $m_{1}$ is the monomer concentration in solution in equilibrium with the pseudophase, that is, CAC.

The standard Gibbs energy of aggregations per mole is, therefore, simply given by the equation:

$$
\Delta_{\mathrm{ag}} G_{n}^{0}=R T \ln (\mathrm{CAC}) .
$$

The results obtained for the thermodynamic functions for the three transfer process are presented in Table 2.

\section{Conclusions}

With respect to the aim of this paper, some general important aspects should be pointed out. First of all, the enthalpy of solution, determined by direct calorimetry allows a detailed insight into solute self-interactions of terfenadine in solution.

Useful conclusions were drawn from the cosolvent systems used. The water plays an important role in the formation the solid phase from the solution. A cooperative self-assembling of the solute molecules giving units of high aggregation number is a characteristics of ethanol/water mixtures. At lower water content the n-mers are formed from monomers. As the water percentage is raised solute stepwise association takes place. From these findings it is very likely that different structural solid forms of terfenadine are obtained using ethanol, ethanol/water and varying the composition of this mixed solvent. 
In the thermodynamic view point aggregation is followed by a small increase of enthalpy but a large increase of entropy. Aggregation is fundamentally an entropy driven process.

\section{References}

[1] T.G. Fawcett et al., US Patent, no. 4742 175, May 3, 1988.

[2] A. Magni, European Patent 0346765 A2, Bulletin 89/51.

[3] J. McCarty, European Patent 0385375 A1, Bulletin 90/36.

[4] J. McCarty, European Patent 0396100 A1, Bulletin 90/45.

[5] J. Canotilho, F.S. Costa, A.T. Sousa, J.S. Redinha, M. Luísa, P. Leitão, Thermochim. Acta 299 (1997) 1.
[6] B. Jönsson, B. Lindman, K. Holmberg, B. Kronberg, Surfactants and Polymers in Aqueous Solutions, ch. 2, 1st ed., Wiley, Chichester, 1998.

[7] J.N. Israelachvili, Intermolecular and Surface Forces, 2nd ed., Part 3, Academic Press, London, 1992.

[8] R. Nagarajan, E. Ruckenstein, Langmuir 7 (1991) 2934.

[9] B.Z. Chowdhry, M.J. Snowden, S.A. Leharne, J. Phys. Chem. B. 101 (1997) 10226.

[10] J. Canotilho, F.G. Costa, A.T. Sousa, J.S. Redinha, M. Luísa, P. Leitão, unpublished Data.

[11] C. Minero, E. Pelizzetti, Pure and Appl. Chem. 65 (1993) 2573.

[12] D. Blankschtein, G.M. Thurston, G.B. Benedek, Phys. Rev. Lett. 54 (1985) 955.

[13] S. Puvvada, D. Blankschtein, J. Chem. Phys. 92 (1990) 3710.

[14] J. Armstrong, B. Chowdhry, R. O'Brien, A. Beezer, S. Mitchell, S. Leharne, J. Phys. Chem. 99 (1995) 4590. 